\title{
Effect of the Positron Distribution on the Electron-Positron Momentum Density Calculated for $\mathrm{SiC}$
}

\author{
A. RubaszeK* \\ W. Trzebiatowski Institute of Low Temperatures and Structure Research \\ Polish Academy of Sciences \\ P.O. Box 1410, 50-950 Wrocław 2, Poland

\begin{abstract}
To interpret positron annihilation data in solids in terms of the electron momentum density, both the electron-positron interaction and positron distribution have to be considered explicitly. In the present work the influence of the shape of the positron wave function on the calculated electron-positron momentum density in elemental $\mathrm{SiC}$ is discussed. It is shown that the form of the positron distribution in the Wigner-Seitz cell has a considerable effect on the resulting annihilation characteristics. Calculations were performed for $\mathrm{SiC}$ of $3 C$ diamond structure within the linear muffin-tin orbital-atomic spheres approximation method.
\end{abstract}

PACS numbers: 78.70.Bj, 71.15.Ap, 71.20.Mq

\section{Introduction}

The increasing interest in the electronic properties of $\mathrm{SiC}$ [1] is mainly due to vital importance of this semiconductor for industry and technology. Here the positron annihilation spectroscopy is a sensitive method to probe the electronic structure of the material under study $[2,3]$. In particular, the angular correlation of annihilation radiation and coincidence Doppler broadening experimental data for $\mathrm{SiC}$ [3] contain useful information on the relevant electron momentum density (EMD), which is an important characteristics of the electron band structure. This information is, however, distorted by the effect of both positron distribution and enhancement of electron density at the positron site, due to strong Coulomb attraction between the positron and surrounding electron cloud. While the resulting change of the electron density at the positron position has been widely studied (see Refs. [2, 4-7] and references cited therein), much less attention has been

*e-mail: A.Rubaszek@int.pan.wroc.pl 
given to the influence of the positron distribution on the calculated annihilation characteristics in solids $[4,5]$.

In the present paper the influence of the electron-positron $(\mathrm{e}-\mathrm{p})$ interaction on the positron charge distribution in the Wigner-Seitz cell is discussed, and the effect of the resulting positron wave function on the positron annihilation characteristics in $\mathrm{SiC}$ is studied.

\section{Calculation}

The $\mathrm{e}-\mathrm{p}$ momentum density is given by the formula

$$
\rho(\boldsymbol{p}=\boldsymbol{k}+\boldsymbol{G})=\sum_{\boldsymbol{k} j}\left|\int \exp (-\mathrm{i} \boldsymbol{p} \cdot \boldsymbol{r}) \psi_{\boldsymbol{k} j}(\boldsymbol{r}) \psi_{+}(\boldsymbol{r}) \sqrt{\gamma_{\boldsymbol{k} j}(\boldsymbol{r})} \mathrm{d} \boldsymbol{r}\right|^{2} .
$$

Here $\boldsymbol{p}$ and $\boldsymbol{k}$ stand for momentum in the extended and reduced zone scheme, respectively, $\boldsymbol{G}$ is the reciprocal lattice vector, $\psi_{\boldsymbol{k} j}$ and $\psi_{+}$denote the electron and positron wave functions, $\gamma_{\boldsymbol{k}_{j}}$ is the e-p correlation function and summation runs over all occupied electron Bloch states $\boldsymbol{k} j$. Within the independent particle model (IPM) the e-p correlation effect is neglected, i.e. one assumes that $\gamma_{\boldsymbol{k} j}(\boldsymbol{r})=1$, and formula (1a) reduces to the expression

$$
\rho^{\mathrm{IPM}}(\boldsymbol{p}=\boldsymbol{k}+\boldsymbol{G})=\sum_{\boldsymbol{k} j}\left|\int_{\Omega} \exp (-\mathrm{i} \boldsymbol{p} \cdot \boldsymbol{r}) \psi_{\boldsymbol{k} j}(\boldsymbol{r}) \psi_{+}(\boldsymbol{r}) \mathrm{d}^{3} \boldsymbol{r}\right|^{2} .
$$

This model seems to be most appropriate for studies of the present work, since the IPM allows to avoid additional complication of the picture, due to the e-p correlation effect.

If the positron is uniformly distributed in the unit cell, i.e. if one assumes that $\left|\psi_{+}(\boldsymbol{r})\right|^{2}=1 / \Omega$, where $\Omega$ is the volume of a primitive cell, then the IPM formula (1b) reduces to the expression which defines the EMD

$$
\rho^{\mathrm{EMD}}(\boldsymbol{p}=\boldsymbol{k}+\boldsymbol{G})=\sum_{\boldsymbol{k} j}\left|(1 / \sqrt{\Omega}) \int_{\Omega} \exp (-\mathrm{i} \boldsymbol{p} \cdot \boldsymbol{r}) \psi_{\boldsymbol{k} j}(\boldsymbol{r}) \mathrm{d}^{3} \boldsymbol{r}\right|^{2} .
$$

The electron and positron wave functions are the solutions of the set of Schrödinger equations,

$$
\begin{aligned}
& {\left[-\nabla^{2}+V_{\mathrm{ext}}(\boldsymbol{r})+V_{\mathrm{H}}(\boldsymbol{r})+V_{\mathrm{xc}}(\boldsymbol{r})\right] \psi_{\boldsymbol{k} j}(\boldsymbol{r})=E_{\boldsymbol{k} j} \psi_{\boldsymbol{k} j}(\boldsymbol{r}),} \\
& {\left[-\nabla^{2}-V_{\mathrm{ext}}(\boldsymbol{r})-V_{\mathrm{H}}(\boldsymbol{r})+V_{\mathrm{corr}}(\boldsymbol{r})\right] \psi_{+}(\boldsymbol{r})=E_{+} \psi_{+}(\boldsymbol{r}) .}
\end{aligned}
$$

The positron Hartree potential and the external potential, due to ions, are equal to the respective electron potentials with the opposite sign, $V_{\mathrm{H}}$, and $V_{\text {ext }}$. $V_{\text {corr }}$ and $V_{\mathrm{xc}}$ denote the e-p interaction $[2,6]$ and e-e exchange-correlation potentials, respectively.

The potential $V_{\text {corr }}$, describing the positron interaction with the electron screening cloud, can be determined from the Feynman theorem $[2,5,6]$. Although several approximations, local or non-local, exist for the $\mathrm{e}-\mathrm{p}$ interaction, in most 
theoretical studies of the positron annihilation characteristics the correlation potential $V_{\text {corr }}$ has been set to zero. The latter approach is due to the IPM [2]. Local density approximation (LDA) [4, 6] to the density functional theory approximates this e-p potential by a local quantity corresponding to the homogeneous electron gas, and therefore is not expected to work well for strongly varying electron densities. For the latter non-local effects will be important, and they can be described, for example within generalized gradient approximation (GGA) [7] or weighted density approximation (WDA) [5].

The calculations of the electron and positron wave functions have been performed for $\mathrm{SiC}$ within the linear muffin-tin orbital-atomic spheres approximation (LMTO-ASA) band structure method [8]. The open diamond structure $3 C$ was considered. This structure is usually modelled as the fcc lattice, containing four atoms per unit cell. For $\mathrm{SiC}$ the two "full" atoms, $\mathrm{Si}$ and $\mathrm{C}$, are located at positions $\boldsymbol{q}_{1}=(0,0,0)$ and $\boldsymbol{q}_{2}=(a / 4, a / 4, a / 4)$, respectively, and two "empty" atoms are at positions $\boldsymbol{q}_{3}=(0,0, a / 2)$ and $\boldsymbol{q}_{4}=(a / 4, a / 4,3 a / 4)$, where $a$ is the lattice constant. The respective average radius of a sphere containing one atom, $S$, is defined in terms of $a$ as $S^{3}=3 a^{3} /(64 \pi)$.

The e-p momentum density was studied within the IPM (formula (1b)) for the positron wave function obtained from the Schrödinger Eq. (2b) within the IPM, LDA, and WDA approaches to $V_{\text {corr }}$. The shell partitioning [5] was applied to the WDA. The non-zero value of the energy gap in $\mathrm{SiC}$ was taken into account through the state-selectivity of the e-p correlation function. Results are compared to EMD (Eq. (1c)), which corresponds to neglecting positron potential in Eq. (2b). The Jarlborg-Singh correction [9] was incorporated in the IPM and EMD formulae (1b) and (1c) in order to account the effect of the atomic spheres overlap.

\section{Results and discussion}

In Fig. 1 it is shown how far the e-p correlation potential influences the positron distribution in $\mathrm{SiC}$, calculated within the ASA. This distribution in spheres containing $\mathrm{Si}$ and $\mathrm{C}$ atoms and in the interstitial region (represented by the empty sphere) is plotted as a function of distance from the sphere centre. IPM, LDA, and WDA denote the approximation used to $V_{\text {corr }}$ in the positron Schrödinger Eq. (2b). Uniform distribution of the positron density in the unit cell is also presented. Comparing the curves one can note that inclusion of the positron interaction with ions and electrons $\left(V_{\text {ext }}\right.$ and $V_{\mathrm{H}}$ in Eq. $\left.(2 \mathrm{~b})\right)$ shifts the weight of the positron wave function from the spheres centred at $\mathrm{C}$ and $\mathrm{Si}$ atoms towards the centre of the empty sphere. This feature is more pronounced for the Si atom (nuclei charge $Z=14$ ) than for the $\mathrm{C}$ atom $(Z=6)$ ). Incorporating the e-p correlation potential reduces this effect, since a "bare" positron is stronger repelled by the ionic cores and attracted by a negative charge of the empty sphere than the screened particle. In consequence, the largest deviation from an uniform distribution occurs for $\psi_{+}^{\mathrm{IPM}}(\boldsymbol{r})$, while the LDA result is intermediate between the 


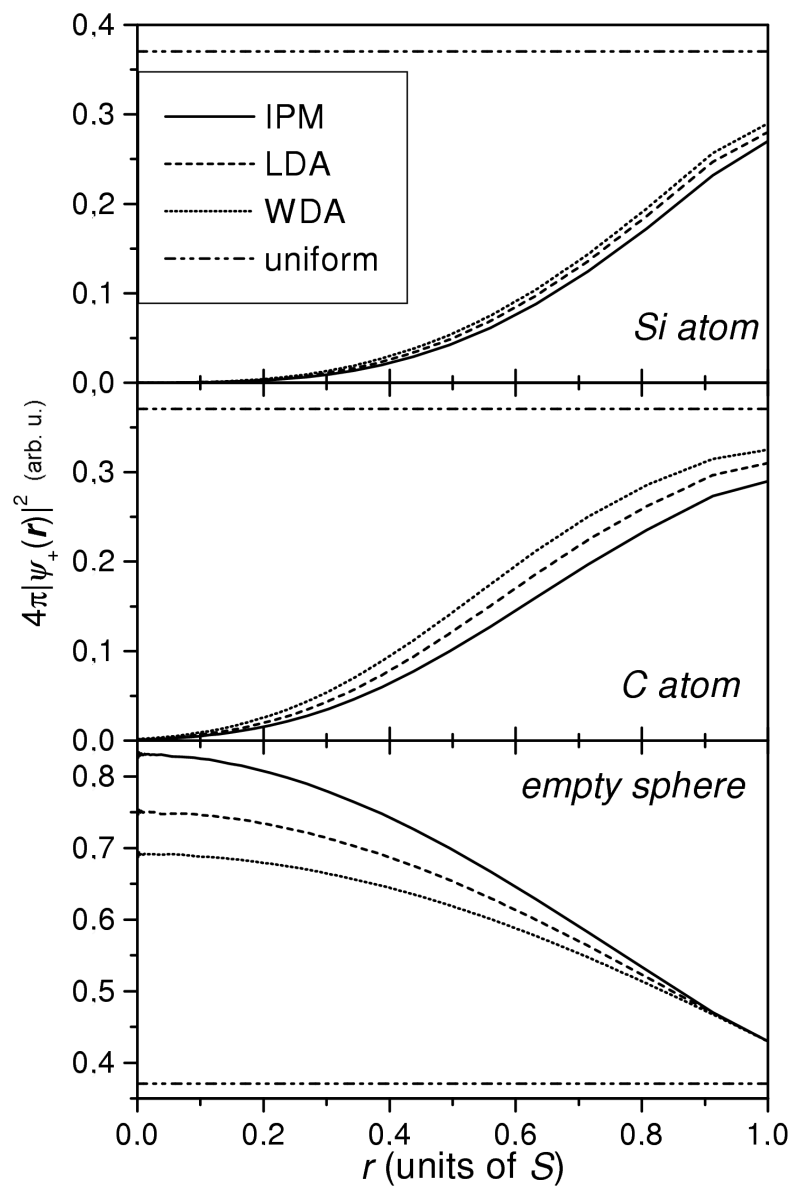

Fig. 1. The positron density distribution as a function of distance from the nuclei calculated for atoms of Si and C (two upper parts) and for the empty sphere (lower part). Solid, dashed and dotted curves represent the IPM, LDA, and WDA results, respectively, and the dash-dotted line corresponds to the uniform distribution, $\left|\psi_{+}(\boldsymbol{r})\right|^{2}=1 / \Omega$.

IPM and non-local WDA distributions. Similar behaviour of $\psi_{+}$is observed in a variety of metals [5].

In Fig. 1 the considerable difference between the positron distribution at the edges of full and empty spheres is well seen. It is worth to mention here that the similar discrepancy is observed in the electron density as well. This occurs not only for $\mathrm{SiC}$, but for other elements of open diamond structure, e.g. silicon and may be attributed to spherical average of the electron (positron) charge, applied within the ASA.

The deviation between LDA and WDA positron densities is mostly pronounced in the empty spheres. This feature may be attributed to the shape 
and localisation of the electron charge, screening a positron. Within the LDA the screening charge distribution is spherically-symmetric around a positron and found at it with highest probability. The WDA screening charge is shifted from a positron towards the region of high electron density inside the $\mathrm{Si}$ and $\mathrm{C}$ spheres. As a result, within the WDA the positron is more "dressed" in the centres of these spheres than within the LDA and therefore less repelled to the interstitial region.

The effect of the positron wave function on the IPM e-p momentum densities, calculated for $\mathrm{SiC}$ along [100] and [111] directions, is illustrated in Fig. 2. It should be noted here that the major part of the electron charge is located inside the spheres containing the $\mathrm{Si}$ and $\mathrm{C}$ atoms. Therefore, the positron redistribution from the uniform density considerably reduces the overlap of the electron and positron wave functions. In consequence, the values of the IPM e-p momentum density, $\rho^{\mathrm{IPM}}(\boldsymbol{p})$, are appreciably diminished with respect to EMD, especially in the low momentum region, as can be well seen in Fig. 2. The properties of IPM, LDA, and WDA positron wave functions are just reflected in relevant slopes of $\rho^{\mathrm{IPM}}(\boldsymbol{p})$. The difference between EMD and $\rho^{\mathrm{IPM}}(\boldsymbol{p})$ is most pronounced for the density, obtained by incorporating $\psi_{+}^{\mathrm{IPM}}$ in the formula (1b). Taking into account the e-p correlation potential in the positron Schrödinger equation increases the relevant momentum density. Non-locality in $V_{\text {corr }}$ enhances this effect even further: application of $\psi_{+}^{\mathrm{LDA}}$ provides results intermediate between those, due to $\psi_{+}^{\mathrm{IPM}}$ and $\psi_{+}^{\mathrm{WDA}}$.

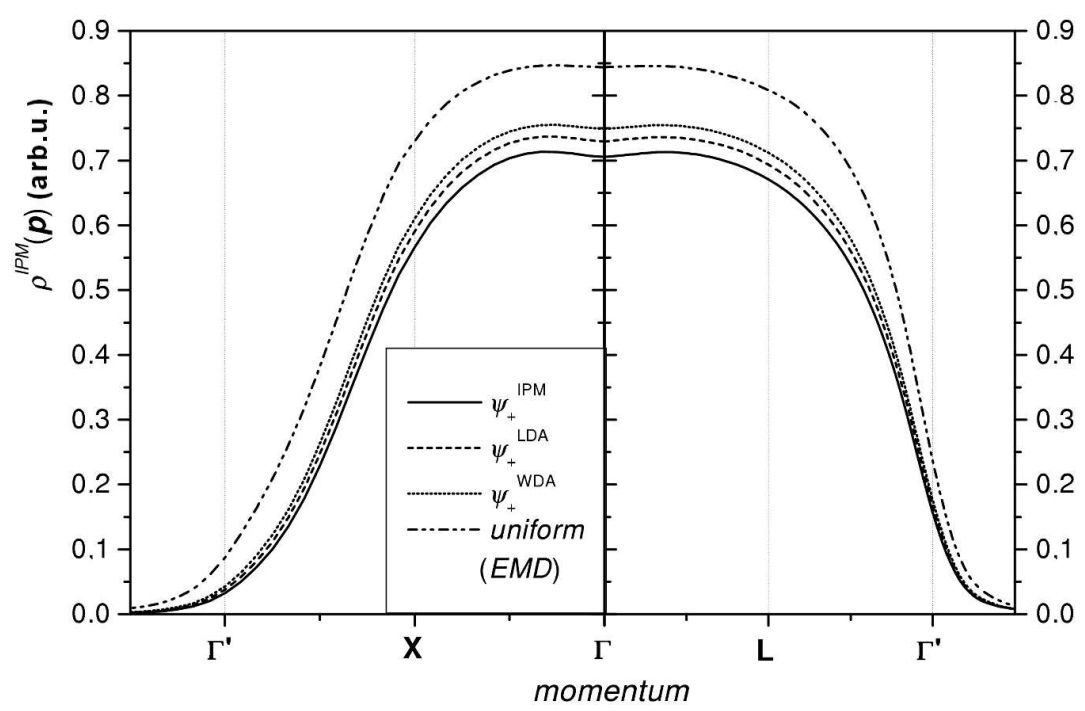

Fig. 2. Electron-positron momentum densities in SiC calculated within the IPM along [100] and [111] directions for $\psi_{+}^{\mathrm{IPM}}, \psi_{+}^{\mathrm{LDA}}$ and $\psi_{+}^{\mathrm{WDA}}$ (solid, dashed and dotted lines, respectively). The electron momentum density, which is due to the uniform positron distribution, is shown by the dash-dotted line. 
Finally, let us note that taking into account the e-p enhancement factor, $\gamma_{\boldsymbol{k} j}(\boldsymbol{r})$, significantly increases values of $\rho(\boldsymbol{p})$ as compared to its IPM counterpart. Non-locality of the e- $\mathrm{p}$ interaction slightly reduces the enhancement effect. Beyond the IPM the picture shown in Fig. 2 changes qualitatively and quantitatively: the EMD is intermediate between $\rho^{\mathrm{LDA}, \mathrm{WDA}}(\boldsymbol{p})$ and $\rho^{\mathrm{IPM}}(\boldsymbol{p})$. Let us note that beyond the IPM both the correlation function $\gamma_{\boldsymbol{k} j}(\boldsymbol{r})$ and the potential $V_{\text {corr }}(\boldsymbol{r})$ have been incorporated to the calculation within the same approximation.

\section{Conclusions}

In a summary one could say that taking into account the interaction of the positron with its screening cloud leads to considerable redistribution of the positron wave function. The weight of the positron density is shifted from the interstitial region towards the atomic cores, as the e- $\mathrm{p}$ correlation potential partially neutralises the repulsive potential of the atomic cores, $-V_{\text {ext }}-V_{\mathrm{H}}$. This effect is enhanced within the non-local approach. The shape of the positron wave function appreciably influences resulting e-p momentum densities for $\mathrm{SiC}$, due to changes in the overlap of the positron and particular electron wave functions.

\section{References}

[1] For review and references see e.g. http://www.ioffe.rssi.ru/SVA/NSM/ Semicond/SiC/bandstr.html.

[2] For review see e.g. M.J. Puska, R.M. Nieminen, Rev. Mod. Phys. 66, 841 (1994); R.M. Nieminen, in: Positron Spectroscopy of Solids, Eds. A. Dupasquier, A.P. Mills, IOS Press, Amsterdam 1996, p. 443; S. Berko, in: Positron Solid State Physics, Eds. W. Brandt, A. Dupasquier, North-Holland, Amsterdam 1983, p. 64: R.M. Nieminen, ibid., p. 105.

[3] A. Kawasuro, M. Yoshikawa, H. Itoh, T. Chiba, T. Higuchi, T. Besuyaku, F. Redmann, R. Krause-Rehberg, Phys. Rev. B 72, 045204 (2005); A. Kawasuro, T. Chiba, T. Higuchi, Phys. Rev. B 71, 193204 (2005).

[4] S. Daniuk, M. Sob, A. Rubaszek, Phys. Rev. B 43, 2580 (1991).

[5] A. Rubaszek, Z. Szotek, W.M. Temmerman, Phys. Rev. B 58, 11285 (1998); ibid., 61, 10100 (2000).

[6] R.M. Nieminen, M.J. Puska, Phys. Rev. Lett. 50, 281 (1983).

[7] B. Barbiellini, M.J. Puska, T. Konhonen, A. Hajru, R.M. Nieminen, Phys. Rev. 53, 1620 (1996).

[8] O.K. Andersen, Phys. Rev. B 12, 3600 (1975); W.R.L. Lambrecht, O.K. Andersen, ibid., 34, 2439 (1986).

[9] T. Jarlborg, A.K. Singh, Phys. Rev. B 36, 4660 (1987). 